Article

\title{
Investigation of Deterministic, Statistical and Parametric NB-PLC Channel Modeling Techniques for Advanced Metering Infrastructure
}

\author{
Bilal Masood ${ }^{1}$, M. Arif Khan ${ }^{2}$, Sobia Baig $\left.{ }^{3}{ }^{(}\right)$, Guobing Song ${ }^{1, *}$, Ateeq Ur Rehman ${ }^{4,5}$, \\ Saif Ur Rehman ${ }^{6}$, Rao M. Asif ${ }^{6}$ and Muhammad Babar Rasheed ${ }^{7}$ (D) \\ 1 School of Electrical Engineering, Xi'an Jiaotong University, Xi'an 710049, China; \\ bilal.masood@mail.xjtu.edu.cn \\ 2 School of Computing and Mathematics, Charles Sturt University, Bathurst, NSW 2678, Australia; \\ mkhan@csu.edu.au \\ 3 Electrical and Computer Engineering Department, COMSATS University Isb- Lahore Campus, \\ Punjab 54000, Pakistan; drsobia@cuilahore.edu.pk \\ 4 College of Internet of Things Engineering, Hohai University, Changzhou 213022, China; \\ ateqrehman@gmail.com \\ 5 Department of Electrical Engineering, Government College University, Lahore 54000, Pakistan \\ 6 Department of Electrical Engineering, The Superior College, Lahore 54000, Pakistan; \\ saifurrehman@superior.edu.pk (S.U.R.); rao.m.asif@superior.edu.pk (R.M.A.) \\ 7 Department of Electronics and Electrical Systems, The University of Lahore, Lahore, Punjab 54000, Pakistan; \\ babarmeher@gmail.com \\ * Correspondence: song.gb@mail.xjtu.edu.cn
}

Received: 18 May 2020; Accepted: 5 June 2020; Published: 15 June 2020

check for updates

\begin{abstract}
This paper is focused on the channel modeling techniques for implementation of narrowband power line communication (NB-PLC) over medium voltage (MV) network for the purpose of advanced metering infrastructure (AMI). Three different types of models, based on deterministic method, statistical method, and network parameters based method are investigated in detail. Transmission line (TL) theory model is used to express the MV network as a two-port network to examine characteristics of sending and receiving NB-PLC signals. Multipath signal propagation model is used to incorporate the effect of multipath signals to determine the NB-PLC transfer function. A Simulink model is proposed which considers the values of MV network to examine the characteristics of NB-PLC signals. Frequency selectivity is also introduced in the impedances to compare variations and characteristics with constant impedances based MV network. A state-of-the-art mechanism for the modeling of capacitive coupling device, and impedances of low voltage (LV) and MV networks is developed. Moreover, a comparative analysis of TL theory and multipath signal propagation models with the proposed Simulink model is presented to validate the performance and accuracy of proposed model. This research work will pave the way to improve the efficiency of next-generation NB-PLC technologies.
\end{abstract}

Keywords: AMI; TL; SG; NB-PLC

\section{Introduction}

A huge challenge faced by the 21st century grid is a large amount of greenhouse gases such as $N O_{X}$ and $C O_{X}$ due to the utilization of fossil fuels in power plants. This dependency of power systems on fossil fuels needs to be reduced to meet the challenges faced by 21 st century grid. The aging of power system infrastructure in most of the countries is another present-day open 
issue [1,2]. The next generation power grid that can cater to these issues is known as smart grid (SG). SG can be regarded as a cyber-physical system that is fully equipped with advanced information and communication technologies (ICT) to efficiently analyze the real-time performance of power grid which will improve the monitoring system, operation, and maintenance of power generation, transmission, and distribution systems. The 21st century ICT can provide various services to SG such as demand side management, demand response, and advanced metering infrastructure (AMI), which will not only facilitate the consumers to act as prosumers but will also reduce the grid reaction time against the power line faults and grid outages. In power line communications (PLC), use of the already existing infrastructure of power systems with cost-effectiveness has gained massive attention from the research community and industry due to the avoidance to install a new costly communication infrastructure. In the present scenario, PLC is certainly an obvious choice to provide SG services [3,4]. The advances and timely updated standardization process of PLC have addressed most of the concerns raised by skeptics. AMI gives an opportunity to the customers to effectively interact with the utilities for real-time electricity pricing and better services with the help of two-way communication. In addition, AMI reduces the power losses and power theft along with remote connect/disconnect of smart meters [5,6]. Despite many advantages of PLC, it still has to go through few inevitable challenges in which the unpredictable channel characteristics of PLC are on top, which makes it very difficult to model the PLC channel. Moreover, the response of PLC channel varies with the variations in the parametric values of PLC channels such as time instant, load/access impedances, and temperature effect. However, its cost-effectiveness and already existing nationwide infrastructure compels researchers and industries to opt for the other choices of communication technologies, i.e., wireless communications [7-9].

PLC is classified into three types: ultra narrowband PLC (UNB-PLC) ranging $0.3-3 \mathrm{kHz}$, narrowband PLC (NB-PLC) with frequency range 3-500 kHz, and broadband PLC (BB-PLC) with frequency range 1.8-250 MHz. This paper uses the NB-PLC frequencies for the investigation of medium voltage (MV) channel characteristics with the help of three different types of techniques. One of the more prominent advantages of NB-PLC is presenting a low attenuation profile at narrowband frequencies (under $500 \mathrm{kHz}$ ) as compared to higher frequencies, especially greater than $1 \mathrm{MHz}$ due to capacitive coupling induced between the earth/ground and power conductor/cable of transmission and distribution system $[1,10,11]$. Such lower attenuations of NB-PLC are helpful in transmitting the AMI data over longer distances, making it a more suitable choice of ICT in SG. The NB-PLC frequency bands are further segregated into four types of European standard CENELEC EN 50065 to provide a better quality of services: (a) CENELEC-A band is operated in 9-95 kHz to provide services by power utilities so that execution of sophisticated monitoring and control of power system can be done; (b) CENELEC-B band is operated in $95-125 \mathrm{kHz}$ and which can be used for any kind application; (c) CENELEC-C band is operated in 125-140 kHz and is dedicated to in-home networking; and (d) CENELEC-D band is operated in 140-148.5 kHz and can be used for alarm and security services. The CENELEC B band and CENELEC D band are unrestricted bands for customers.

The most suitable technology to incorporate the NB-PLC is OFDM, for which international standards such as PRIME, G3-PLC, and IEEE1901.2 are available. PRIME was the first effort made, in 2007, towards the standardization process of next generation PLC technologies with the help of DSO European region development fund (ERDF). The G3-PLC was an initiation of an alliance of 12 companies in 2011 whose main sponsorship was given by ERDF. IEEE Communication Society has also standardized the OFDM based NB-PLC system due to high level of interest shown by industries [12-14]. NB-PLC technologies also present low data rate (LDR) and high data rate (HDR) NB-PLC services. A few kbps data rate is offered by single carrier NB-PLC techniques, whereas, for HDR services, having an ability to transmit a large amount of data up to 100s of kbps can be achieved by multicarrier modulation techniques. The NB-PLC technologies operated at LDR incorporates the standards ISO/IEC (14908-3, 14543-3-5), IEC (61334-3-1, 61334-5), CEA-600.31, etc. 


\subsection{Mian Contribution}

This paper proposes a sophisticated channel modeling technique for the investigation of narrowband frequencies ( $3-500 \mathrm{kHz}$ ) over MV network. Three different types of channel modeling techniques are presented in this paper for the comparative analyses and to examine the performance of proposed Simulink model: (1) Transmission line (TL) theory model, which is a deterministic modeling approach, is used in which constant and frequency selective (FS) low voltage LV and MV networks are taken into account. (2) Multipath signal propagation model based on statistical approach is used to examine the characteristics of NB-PLC signals by considering the effect of multipath signals. (3) The proposed Simulink model incorporates the values of network parameters that is to be investigated. Impedances are modeled by three types of impedance modeling techniques, formulated by the combination of series and parallel resonant circuits. A comparative analysis of obtained transfer functions is presented to validate the accuracy of proposed Simulink model. This research work facilitates the electric supply companies and researchers to examine the NB-PLC performance over MV network by simply incorporating the parameters of network under evaluation, instead of carrying out extensive and time taking field measurements. The analysis of MV NB-PLC network helps to assess the feasibility for the implementation of advanced metering infrastructure (AMI) using NB-PLC technologies.

\subsection{Paper Structure}

The paper is divided into seven sections. Section 2 discuss the related work and literature on PLC channels and their modeling techniques. Section 3 gives an overview on the characteristics of MV NB-PLC network. Section 4 characterizes the MV NB-PLC network by focusing on TL parameters such as resistance, conductance, inductance, capacitance, and characteristics impedance. Resistance variations law and impedance modeling is examined in depth by discussing their results. Section 5 elaborates various channel modeling techniques and proposed a Simulink model to compute the transfer function of MV network to analyze the performance of NB-PLC. Section 6 discusses and compares the results obtained from TL theory and multipath signal propagation models with Simulink model. This is followed by the conclusions in Section 7.

\section{Related Work}

Stefano Galli, a leading researcher in the field of deterministic PLC channel modeling, has made valuable contributions in the area of indoor PLC channels [14,15]. In his model, Galli addressed indoor PLC problems, using transmission lines theory, primarily focusing on model decomposition and multiconductors. The transmission lines theory analyzed propagation interaction of two coupled circuits and dominant modes: differential and pair modes. The differential propagation of signals is investigated by the differential mode while the pair mode's excitation and propagation are studied by the companion model. In [16], the author emphasized that, on the one hand, the best way to achieve a comprehensive and efficient PLC is to use the differential and companion models as a cascaded two-port network by employing transmission matrix techniques, while, on the other hand, neglecting the mode coupling and companion circuit leads to an incomplete circuit model that is unsuitable for signal propagation of PLC. He eventually came up with a single lumped network that has the ability to replace distributed parameters of circuits. He claimed experimentally and mathematically that, regardless of its topology, the transfer functions for indoor PLC remain the same. He also provided experimental evidence to show that the resonant modes and reflections can be isolated depending on their specific topologies [14].

Time-varying properties of the power line vary due to the following two factors [17]: (1) the layout of topology; and (2) the switching of devices and appliances installed indoors. These continuous varying impedances coupled with instantaneous amplitude of the mains voltage give rise to a periodically time-varying channel response. In $[18,19]$, the authors mainly benefited from Galli's 
methodology with the only exception of using a different conductor scheme. This PLC model thus employed two-conductor and multi-conductor concepts. Andrea M. Tonello employed a random topology technique using statistical bottom-up approach to study PLC channel modeling, whereby he produced transfer function by utilizing computational efficiencies [20]. First, Tonello studied European in-home network topology by applying statistical methods. Second, he determined transfer function between any pair of outlets of a given topology in a unique way. Later, to study PLC channel characteristics statistically, he also developed a simulator to configure a small set of parameters for theoretical frameworks [21,22].

The authors of [23] conducted a comprehensive field trial over MV overhead power lines in which signal passes through a MV/LV transformer bridge. A simulation model to examine the PLC characteristics was also modeled. A low voltage, high frequency PLC signal was injected into the MV bus bar to cross the transformer bridge and received at LV side of transformer. The suggested PLC model was an effective tool to simulate the MV network by focusing on MV power line channel modeling. Field measurements were performed on Favignana Island on a core-shield type conductor configuration for the PLC communication over it. The measured and simulated results were compared to validate the performance of simulated model $[23,24]$. A line-shielded MV network with power transformers, couplers, transmitter, and receiver system was tested for diverse range of line lengths. Zimmermann [9] was among the first researchers to incorporate the physical effects of PLC channel by considering the multipath signals, losses in cable, and time delays when the PLC signal propagates throughout the length of power line. A complex transfer function of PLC channel was statistically investigated in depth to access the feasibility of PLC systems. The proposed multipath signal propagation model uses the parameters of power line; however, it provides a complex frequency response to study the PLC channels characteristics. The model employed the frequency range between $500 \mathrm{kHz}$ and $20 \mathrm{MHz}$, providing the transfer function, although it is not completely familiar with the exact values of network parameters. This PLC signal of this model is an accumulation of all propagated signals in all possible paths while traveling towards the destination [10-12]. The identification of transfer functions is necessary and based on measured values. Table 1 summarizes the contributions of authors for various types of PLC models. For the characterization of power line channels, modeling of their parameters is very important. Detailed discussion on power line parameters for the purpose of channel modeling can be found in [25-27]. In [25], the voltage-current approach is applied to determine access impedance. The shunt resistance $R_{s h}$ is used as a parameter to measure the current. Impedance modeling is expressed as,

$$
Z_{a, k}=Z_{m, k}-\left(R+j \omega L+\frac{1}{j \omega C}\right)
$$

where $1 \phi$ impedance at reference point $B$ is denoted by $Z_{a, k}$, which can be calculated by measured values of impedance $Z_{m, k}$, that is $1 \phi$ impedance at reference point $A$.

$$
Z_{a, 3-p h}=Z_{m, 3-p h}-Z_{3-p h a s e / c a l i b r a t i o n}
$$

where the $3 \phi$ coupling impedance at reference point $B$ is denoted by $Z_{a, 3-p h}$ determined by measured three-phase impedance $Z_{m, 3-p h}$ at $A$ reference point. The calibration impedance that determines the coupling network impedance is denoted by $Z_{3-p h a s e / c a l i b r a t i o n}$. The calculation to obtain the access impedance theoretically can be achieved by parallel combination of $1 \phi$ impedances located at each phase by,

$$
Z_{a, 3-p h-\text { theoratical }}=\frac{1}{\frac{1}{Z_{a, 1}}+\frac{1}{Z_{a, 2}}+\frac{1}{Z_{a, 3}}}
$$


Canete et al. [27] proposed an indoor PLC channel model. Three different types of load impedances i.e., FS, constant and time varying were taken into account. The FS impedance are modeled by,

$$
Z_{\omega}=\frac{R}{1+j Q\left(\frac{\omega}{\omega_{0}}-\frac{\omega_{0}}{\omega}\right)}
$$

where $R, Q$, and $\omega_{0}$ represent the resonant resistance, quality factor, and resonance angular frequency, respectively.

The time-varying impedances are modeled by,

$$
Z_{\omega, t}=Z_{A}(\omega)+Z_{B}(\omega)\left|\sin \left(\frac{2 \pi}{T_{0}} t+\phi\right)\right| ; 0 \leq t \leq T_{0}
$$

where $Z_{A}, Z_{B}$, and $\phi$ are offset impedance, amplitude variation, and phase, respectively.

Table 1. Summary of research work contributions in various types of PLC models.

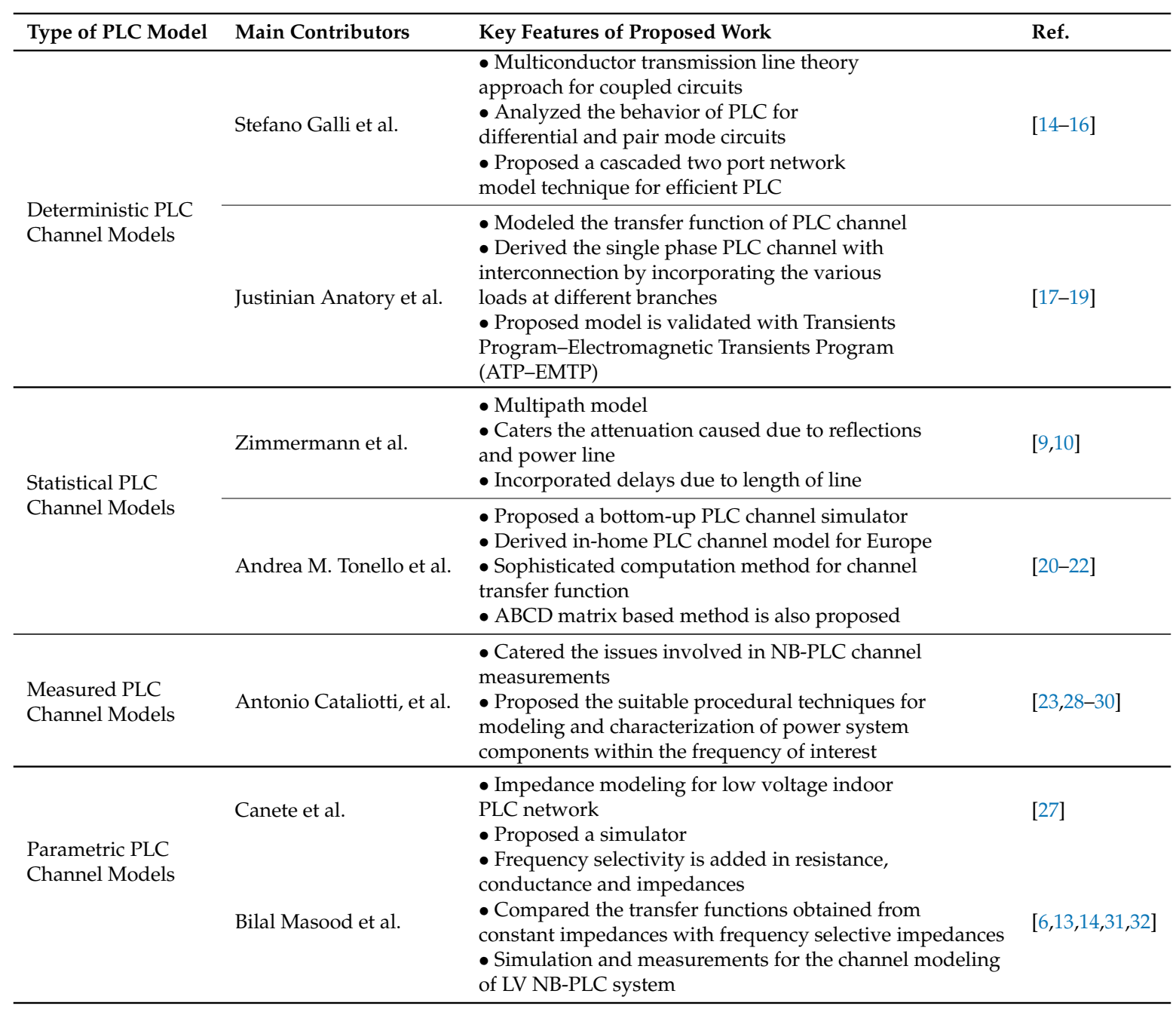

\section{Channel Modeling of NB-PLC for AMI in Medium Voltage Network}

One of the challenges faced by NB-PLC-based AMI system is the inimical characteristics of MV channel [28-30,33,34]. A model of NB-PLC system for the purpose of AMI is depicted in Figure 1. The performance of NB-PLC-based AMI system is poorly influenced by various factors such as multipaths, time dispersion, reflections, propagation and time delay, and FS. When the signal is propagated in MV channel, it has to go through issues such as phase shift replication, delay, 
and attenuation. The root mean square technique can be used to cater the time dispersion issues during measurement process. Few problems such as dispersion and time selectivity are common in both types of channels, i.e., wireless and NB-PLC. Furthermore, consideration of additive white Gaussian noise cannot be taken as a reference to completely understand the attributes of NB-PLC channel $[16,35,36]$. There is an utmost need to devise a criterion for the modeling of MV NB-PLC channels that can serve to examine the NB-PLC system for various types of noises. PLC systems are usually affected by two types of noises, i.e., background noise and impulsive noise, as shown in Figure 2 [10]. Their further classifications are: (a) impulsive noise is a combination of apriodic impulsive noise with asynchronous or synchronous impulsive noise; and (b) background noise consists of colored noise and narrowband noise. In this paper, a Simulink model is proposed for the characterization and modeling of MV NB-PLC channels for the purpose of AMI. The real-time overhead MV transmission lines are used in the proposed model Simulink model. A CCD helps to inject the NB-PLC signal in the MV power line, the working principle of which is discussed in Section 5.3.1. A comparison of transfer functions computed from Simulink model are compared with TL theory (constant and FS) and multipath signal propagation model to validate the accuracy of proposed Simulink model. Characterization of MV NB-PLC network is discussed in the subsequent section.

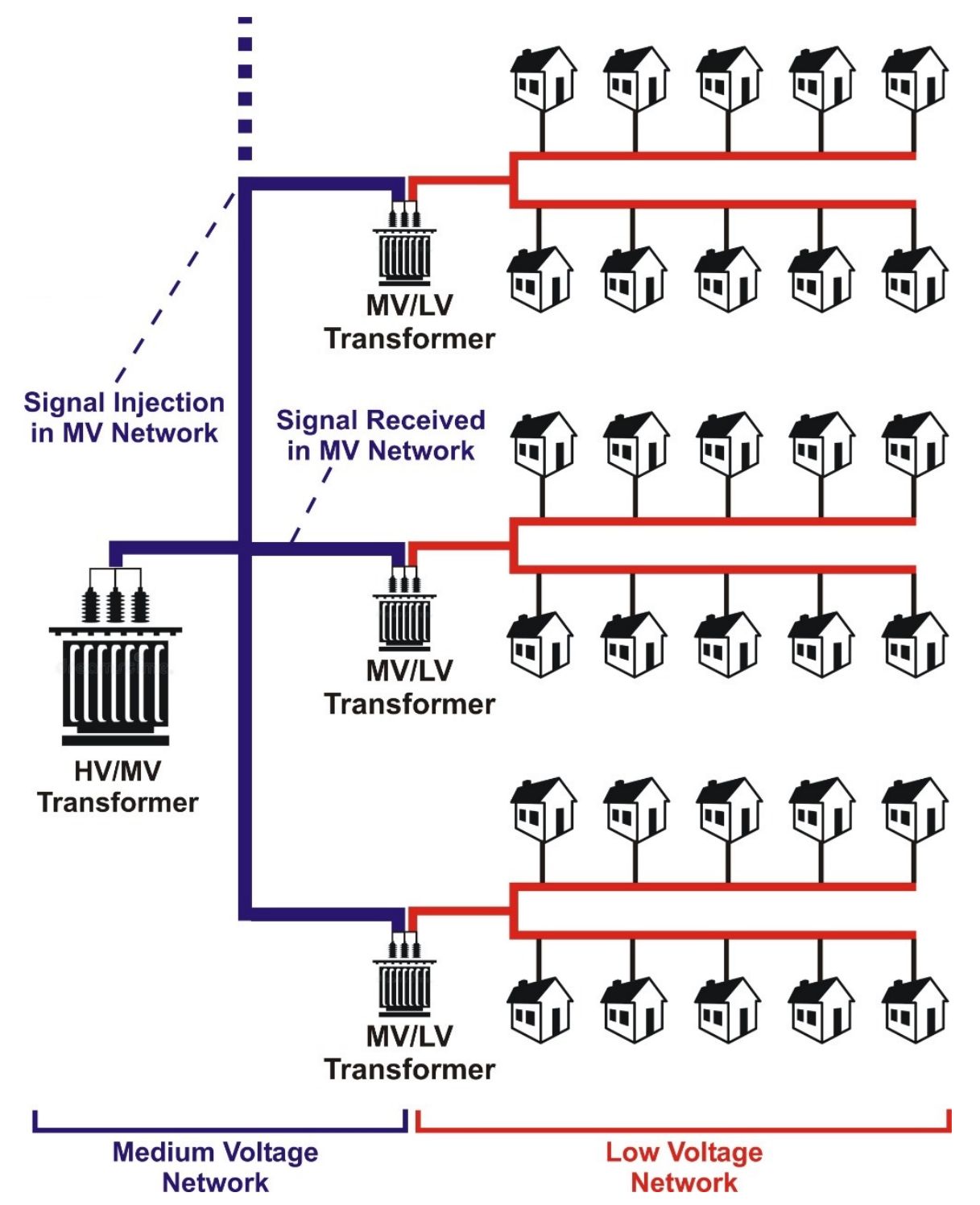

Figure 1. NB-PLC model for MV network. 


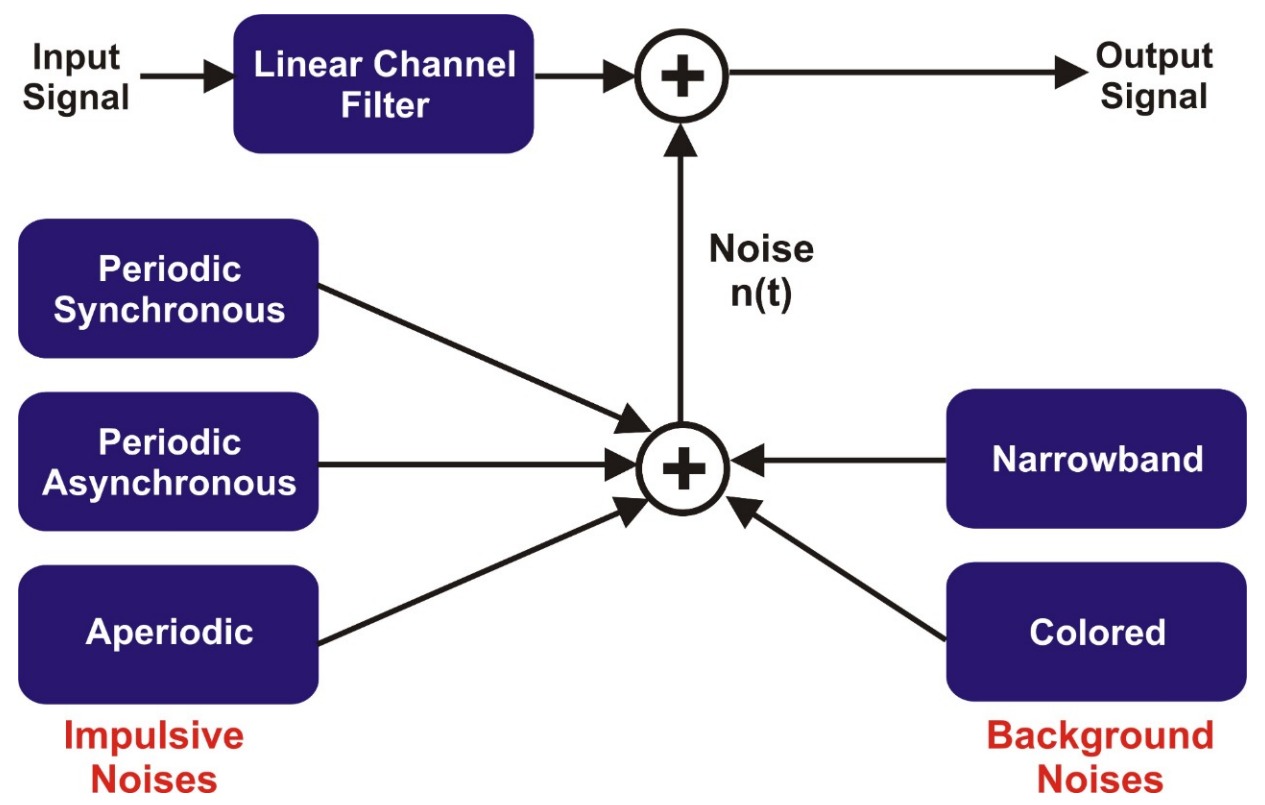

Figure 2. Noise scenario in power line communication system.

\section{Characterization of Medium Voltage NB-PLC Network}

This section deals with the modeling of MV transmission line. The electrical parameters of actual transmission lines are tabulated in Table 2. The conductor types Ant BS 215 and Lynex BS 215 are used for LV and MV network with ampacity up to 175 and $384 \mathrm{~A}$, respectively. Ant has an all aluminium conductor (AAC) type, whereas Lynex has aluminium conductors steel reinforced (ACSR) type with a steel reinforcement to increase the tensile strength due to its weight. The characteristics of any transmission line can be analyzed by its distributed parameters such as resistance $R$, conductance $G$, capacitance $C$, and inductance $L$. These parameters in the case of overhead transmission lines can be determined as [23],

$$
\begin{gathered}
R=\frac{1}{\pi a} \sqrt{\frac{\pi f \mu}{\sigma}}[\Omega] \\
G=2 \pi f C \tan \delta[S] \\
C=\frac{\pi \varepsilon}{\cosh ^{-1}\left(\frac{D}{2 a}\right)}[F / m] \\
L=\frac{\mu}{\pi} \cosh ^{-1}\left(\frac{D}{2 a}\right)[H / m]
\end{gathered}
$$

where $\varepsilon, \mu, \delta, D$, and $\sigma$ are permittivity of free space, permeability of free space, depth factor, diameter of conductor, and conductivity of material, respectively. The frequency of inters in this paper ranges 3-500 kHz. However, the characteristic impedance $Z_{C}$ and propagation constant $\gamma$ of transmission line can be calculated as,

$$
\begin{gathered}
Z_{C}=\sqrt{(R+j \omega L) /(G+j \omega C)} \\
\gamma=\alpha+j \beta=\sqrt{(R+j \omega L)(G+j \omega C)}
\end{gathered}
$$

where attenuation constant is denoted by $\alpha$, whereas $\beta$ denotes the phase factor and $\omega$ represents the angular frequency. 
Table 2. Electrical parameters of conductors.

\begin{tabular}{lllllllll}
\hline $\begin{array}{l}\text { Conductor } \\
\text { Type/Standard }\end{array}$ & $\begin{array}{l}\text { Nominal/ } \\
\text { Section Area }\end{array}$ & $\begin{array}{l}\text { No./Nominal } \\
\text { Diameter of Wires }\end{array}$ & $\begin{array}{l}\text { Approximate Overall } \\
\text { Diameter }\end{array}$ & $\begin{array}{l}\text { Nominal DC } \\
\text { Resistance at 20 C }\end{array}$ & Current Rating & L & C \\
\hline mm & $\begin{array}{l}\text { Aluminium } \\
\mathbf{( N o . / m m}\end{array}$ & $\begin{array}{l}\text { Steel } \\
\mathbf{( N o . / m m})\end{array}$ & $\mathbf{m m}$ & $\mathbf{\Omega} / \mathbf{k m}$ & Amps & $\boldsymbol{\mu H} / \mathbf{m}$ & $\mathbf{p F / m}$ \\
\hline Ant-BS 215 & 52.8 & $7 / 3.10$ & - & 9.30 & 0.54190 & 175 & 0.93 & 12.6 \\
\hline Wolf BS 215 & 212.10 & $30 / 2.59$ & $7 / 2.5$ & 18.13 & 0.1828 & 351 & 1.22 & 9.45 \\
\hline Lynex BS 215 & 226.2 & $30 / 2.79$ & $7 / 2.7$ & 19.53 & 0.1576 & 384 & 1.20 & 9.58 \\
\hline Panther BS 215 & 261.5 & $30 / 3.0$ & $7 / 3$ & 21.0 & 0.1363 & 420 & 1.15 & 9.8 \\
\hline
\end{tabular}

\subsection{Resistance Variations Law}

Resistance variations law is used to characterize the variations in transmission line's resistance with respect to change in frequency [23]. Frequency selectivity of resistance using resistance variations law can be calculated as,

$$
R(f)=A_{R} f^{2}+B_{R} f+C_{R}
$$

where the values of $A_{R}, B_{R}$ and $C_{R}$ are $2.5 \times 10^{-10} \mathrm{~m} \Omega /\left(\mathrm{m}^{*} \mathrm{kHz}{ }^{2}\right), 1.5 \times 10^{-12} \mathrm{~m} \Omega / \mathrm{m}^{*} \mathrm{kHz}$ and $3 \mathrm{~m} \Omega / \mathrm{m}$, respectively. Figure 3 compares the resistance variations with respect to increase in frequency obtained from Equation (6) and resistance variation law. The plotted results depict that variations in the values of resistance calculated from resistance variations law are close to the FS resistance values that validate the simulation results. Since the MV network under evaluation incorporates overhead transmission lines where the separation medium between two lines is free space, the conductance is assumed to be zero.

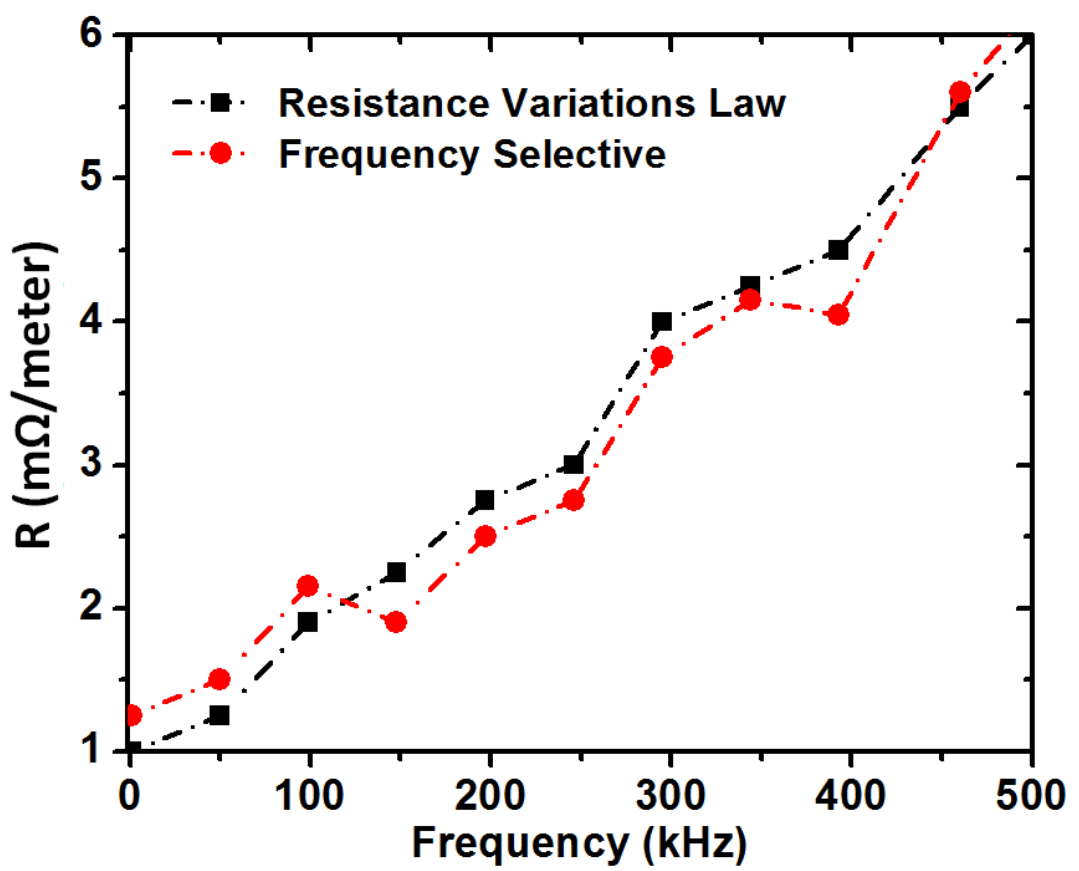

Figure 3. Comparison of FS resistance with the resistance obtained from resistance variations law.

\subsection{Modeling of Impedances for Medium Voltage NB-PLC Network}

Impedance modeling plays a vital role in NB-PLC systems that behave as hurdles for injected signals in power lines. Line impedances with lower values can cause a high level of attenuation to the transmitted signal, carrying high frequency with a small magnitude of injected signal. In practical situation, it becomes a challenge for field engineers to inject a signal in MV NB-PLC channels which have impedance values lower than $0.5 \Omega$. It is also important to note that access impedances are FS which vary with the change in frequency. In [25-27], a comprehensive discussion on the 
characterization and modeling can be found. Chu et al. [25] gave an overview of the LV network in the context of examining the access impedances. However, by distributing the noise over frequency range $50-500 \mathrm{kHz}$, characteristics of LV access impedances are investigated in [26].

It is worth mentioning that TL theory-based transfer functions in this paper comprise of two types:

- Constant LV and MV Networks: The constant LV and MV network includes the fixed values of transmission lines and access impedances parameters.

- FS LV and MV Network: The FS parameters includes the values of transmission lines and access impedances that varies with an increase of frequency of NB-PLC signal.

Three types of impedance modeling methods are used in this paper, as illustrated in Figure 4. The reason behind choosing the combination of parallel and series resonant circuits is critical access impedances connected to LV and MV power line channels carry the resonant behavior that can be achieved by such combination of circuits [6]. This paper incorporates Types 1,2, and 3 for the purpose of impedance modeling in the TL theory method and Simulink model. The formulation of parallel and series combination of two resonant circuits is given as,

$$
\begin{aligned}
& Z_{S}(f)=\frac{1+j 2 \pi R_{S} C_{S}+(j 2 \pi f)^{2} L_{S} C_{S}}{2 \pi f C_{S}} \\
& Z_{P}(f)=\frac{R_{P}+j 2 \pi f L_{P}}{1+j 2 \pi R_{P} C_{P}+(j 2 \pi f)^{2} L_{P} C_{P}}
\end{aligned}
$$

where the subscript $S$ denotes the series circuits and subscript $P$ represents the parallel circuits.

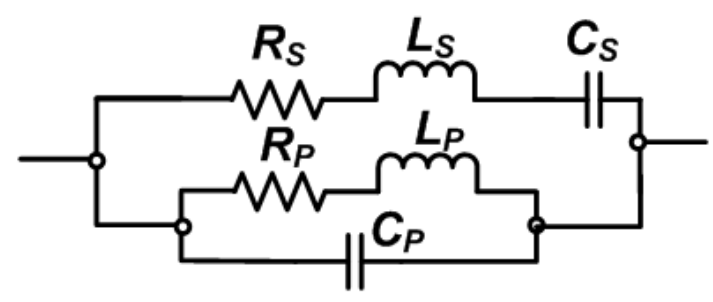

(a)
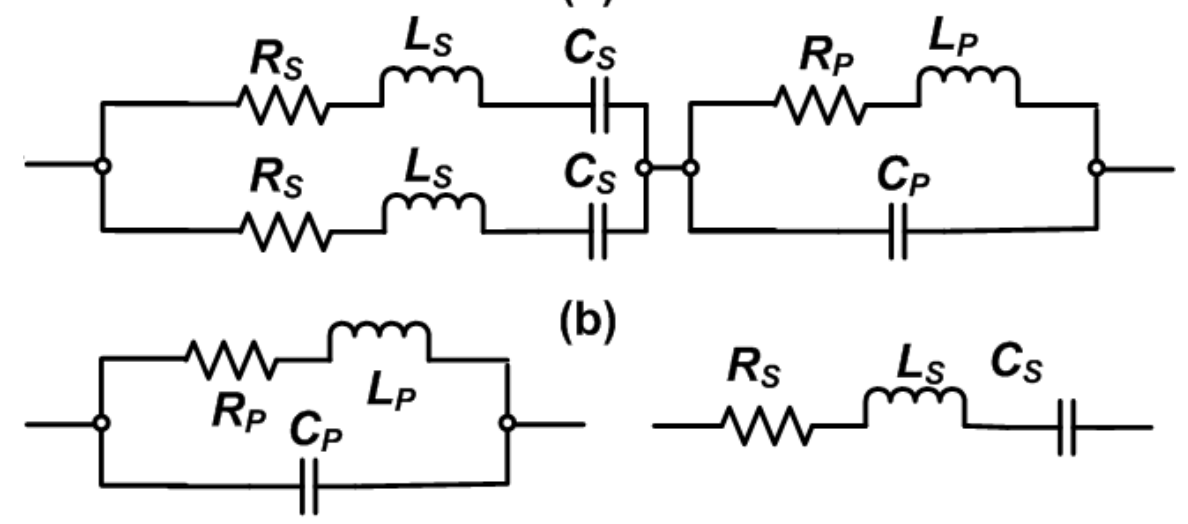

(c)

Figure 4. Modeling of access impedances by resonant circuits: (a) Type 1; (b) Type 2; and (c) Type 3.

\subsubsection{Type 1 Circuit}

By using Equations (13) and (14), Type 1 circuit is modeled by the combination of two RLC resonant circuits connected in series and parallel given as,

$$
Z_{r 1, r 2}(f)=\frac{Z_{S}(f) Z_{P}(f)}{Z_{S}(f)+Z_{P}(f)}
$$




\subsubsection{Type 2 Circuit}

The Type 2 circuit is a combination of three resonant circuits in which two series and one parallel combination of RLC elements are included given as,

$$
Z_{r 3}(f)=\frac{Z_{S 1}(f) Z_{S 2}(f)}{Z_{S 1}(f)+Z_{S 2}(f)}+Z_{P}(f)
$$

\subsubsection{Type 3 Circuit}

The simplest case for analysis could be a series or parallel resonant circuit that is expressed in Type 3.

\subsubsection{Input Impedance of MV Network}

The $Z_{\text {in }}$ (equivalent input impedance) of MV network is determined as,

$$
Z_{\text {in }}=Z_{c b t} \frac{Z_{b t}+Z_{c b t} \tanh \left(\gamma_{b t} l_{b t}\right)}{Z_{c b t}+Z_{b t} \tanh \left(\gamma_{b t} l_{b t}\right.}
$$

where $Z_{b t}$ denotes the bridge tap impedance connected with LV and MV networks, $Z_{c b t}$ is bridge tap characteristic impedance, $l_{b t}$ represents the bridge tap length, and $\gamma_{b t}$ is a propagation constant of bridge tap.

\subsection{Discussion of Results on Impedance Variations of NB-PLC Network}

Figure 5 elaborates the characteristics of impedance variations in NB-PLC network by incorporating both constant and FS types of impedances. The purpose of investigating both impedance types is to analyze whether the impedances vary more in the case of FS as compared to constant and then to compare their transfer functions for NB-PLC channel by TL theory model (constant and FS) and Simulink model in the next subsection.
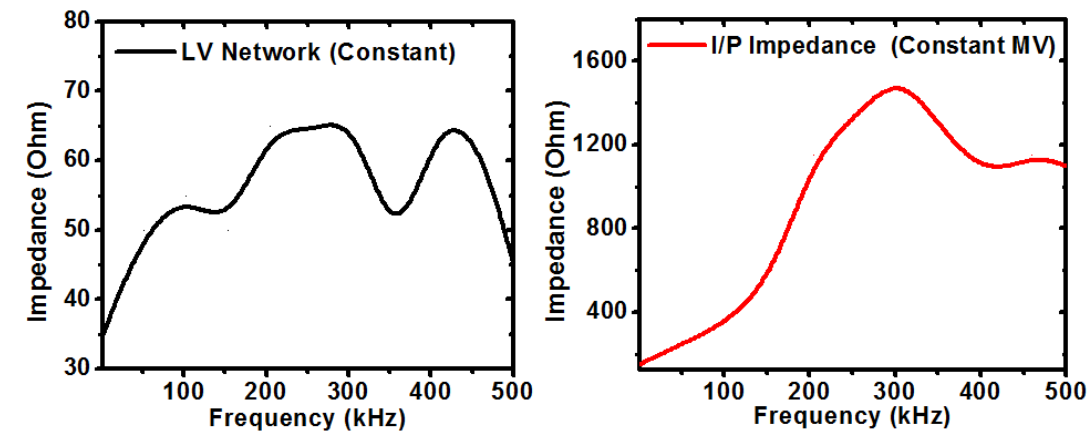

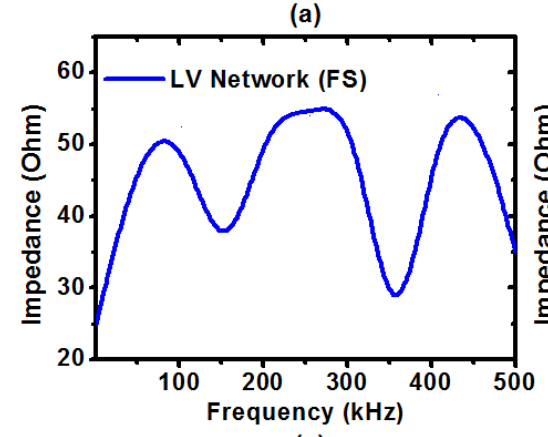

(c)

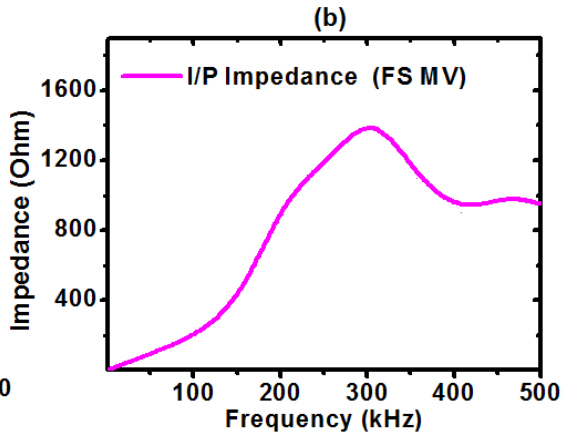

(d)

Figure 5. Impedance variations of LV and MV Networks: (a) constant LV network; (b) input impedance of constant MV network; (c) frequency selective LV network; and (d) input impedance of frequency selective MV network. 


\subsubsection{Discussion on Constant NB-PLC Network Impedances}

Figure $5 \mathrm{a}, \mathrm{b}$ illustrates the impedance variations by considering the constant impedances. The constant LV network connected with MV/LV transformer is shown in Figure 5a, whereas Figure 5b depicts the input impedance of complete MV network while considering the constant LV network. It is noteworthy that the impedance values of loads connected to main channels such as LV network connected to MV (main NB-PLC channel) vary within 35-65 $\Omega$.

\subsubsection{Discussion on Frequency Selective LV and MV Network Impedances}

Figure $5 c$,d illustrates the impedance variations by considering the FS impedances. The FS LV network connected with MV/LV transformer is shown in Figure $5 c$, whereas Figure $5 d$ depicts the input impedance of complete MV network while considering the FS LV network. When comparing the plots obtained by using constant impedances with FS impedances, it can be seen that the FS impedances-based plots show more variations in the magnitudes as compared to constant impedances based plots. In the later sections of this paper, the effect of constant and FS impedances on transfer functions is discussed as well as compared with the proposed Simulink model.

\section{Methodologies to Determine NB-PLC Transfer Functions}

\subsection{Transmission Line Theory}

The TL theory model is incorporated to determine the MV power line channel transfer function. According to TL theory, power network can be expressed by the ABCD matrix that formulates a relation between sending end current $I_{1}$ and voltage $V_{1}$ with the receiving end current $I_{2}$ and voltage $V_{2}$ given as,

$$
\left[\begin{array}{c}
V_{1} \\
I_{1}
\end{array}\right]=\left[\begin{array}{cc}
\cosh (\gamma l) & Z_{c} \sinh (\gamma l) \\
\frac{1}{Z_{c}} \sinh (\gamma l) & \cosh (\gamma l)
\end{array}\right]\left[\begin{array}{c}
V_{2} \\
I_{2}
\end{array}\right]
$$

The subsections $T_{0}$ (series impedance as a two port network), $T_{1}$ (power lines as a two port network), $T_{2}$ (parallel impedance as a two port network), and $T_{3}$ (power lines as a two port network) shown in Figure 6 can be given ABCD matrices form as,

$$
\begin{gathered}
T_{0}=\left[\begin{array}{cc}
1 & Z_{S} \\
0 & 1
\end{array}\right] \\
T_{1}=\left[\begin{array}{cc}
\cosh \left(\gamma_{1} l_{1}\right) & Z_{c l 1} \sinh \left(\gamma_{1} l_{1}\right) \\
\frac{1}{Z_{c l 1}} \sinh \left(\gamma_{1} l_{1}\right) & \cosh \left(\gamma_{1} l_{1}\right)
\end{array}\right] \\
T_{2}=\left[\begin{array}{cc}
1 & 0 \\
\frac{1}{Z_{i n}} & 1
\end{array}\right] \\
T_{3}=\left[\begin{array}{cc}
\cosh \left(\gamma_{2} l_{2}\right) & Z_{c l 2} \sinh \left(\gamma_{2} l_{2}\right) \\
\frac{1}{Z_{c l}} \sinh \left(\gamma_{2} l_{2}\right) & \cosh \left(\gamma_{2} l_{2}\right)
\end{array}\right]
\end{gathered}
$$

where $\gamma_{1}, Z_{c l 1}, \gamma_{2}$, and $Z_{c l 2}$ represent the propagation constants and characteristic impedances of sub-networks, whereas $Z_{i n}$ is input impedance. All of above ABCD matrices are multiplied with each other by chain rule, i.e., a generalized expression for $i$ cascaded sections is given as,

$$
T=\prod_{i=1}^{N} T_{i}
$$




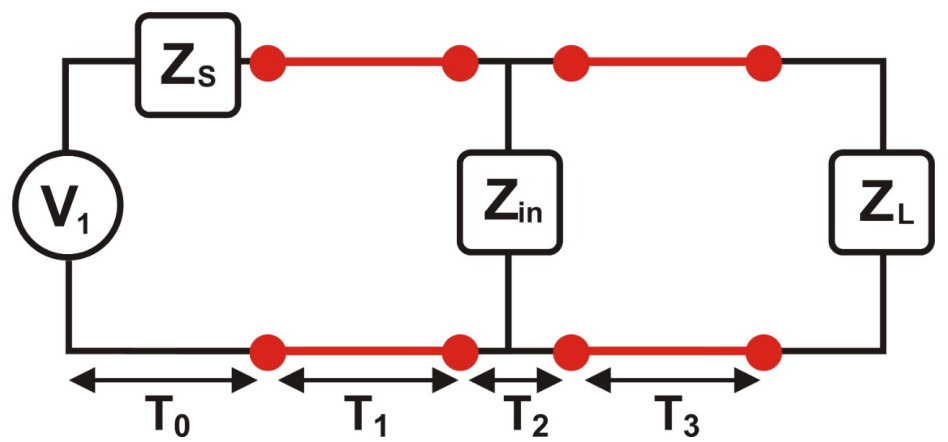

Figure 6. Equivalent transmission network of transmission line with single connection of bridge tap [1].

Finally, transfer function is determined by,

$$
H=20 \log _{10} \frac{Z_{L}}{A Z_{L}+B+C Z_{L} Z_{S}+D Z_{S}}
$$

where $Z_{S}$ and $Z_{L}$ are source and load impedances.

\subsection{Multipath Signal Propagation Model}

The signal propagation model that is based on the signals transmitted and reflected from multiple paths uses the line of sight path for all instants of times [9]. When the signal propagates from transmitter to receiver, a few extra signals, which are known as multipaths, are superimposed and thus added, which is a leading cause of reflections and echos that need to be rectified. The resultant of such scenario is FS fading that degrades the quality of NB-PLC system. Transfer function determined in Zimmermann model is given as,

$$
H(f)=\sum_{i=1}^{n}\left|g_{i}(f)\right| e^{\varphi g_{i}(f)} e^{-\left(a_{0}+a_{1} f^{k}\right) d_{i}} e^{-j 2 \pi f \tau_{i}}
$$

where attenuation, delay, and weighting factor are denoted by $e^{-\left(a_{0}+a_{1} f^{k}\right) d_{i}}, e^{-j 2 \pi f \tau_{i}}$, and $\left|g_{i}(f)\right| e^{\varphi g_{i}(f)}$, respectively. It can be examined by the above-mentioned equation that, when a signal propagates, its attenuation increases with an increase of the length of conductor. Moreover, the response of system reflects the low pass characteristics on NB-PLC frequency range, i.e., 3-500 kHz. The characteristics of NB-PLC signals in regard to reflection and propagation are linked with the weighting factor $g_{i}$. Signal characteristics can be analyzed in general, frequency dependent, and complex form by multipath signal propagation model. This model suggests that $N$ multipaths are added when the signal propagates towards the receiver side and vice versa. The simplified expression of transfer function is expressed as,

$$
H(f)=\sum_{i=1}^{n} g_{i} e^{-\left(a_{0}+a_{1} f^{k}\right) d_{i}} e^{-j 2 \pi f \frac{d_{i}}{v p}}
$$

The parametric values of multipaths are tabulated in Table 3.

Table 3. Parameters of multipath signal propagation model.

\begin{tabular}{cccccc}
\hline \multicolumn{6}{c}{ Parametric Values of Attenuation } \\
\hline$k=1$ & $a_{0}=0$ & $a_{1}=7.8 * 10^{-10} \mathrm{~S} / \mathrm{m}$ \\
\hline \multicolumn{6}{c}{ Path Parameters } \\
\hline $\mathbf{i}$ & $g_{i}$ & $d_{i} / m$ & $\mathbf{i}$ & $g_{i}$ & $\boldsymbol{d}_{i} / \boldsymbol{m}$ \\
\hline 1 & 0.70 & 750 & 3 & -0.20 & 200 \\
\hline 2 & 0.35 & 1000 & 4 & 0.06 & 225 \\
\hline
\end{tabular}




\subsection{Proposed Simulink Model for Medium Voltage NB-PLC Network}

In this section, the proposed Simulink model for the channel modeling and characterization of MV NB-PLC, as shown in Figure 7, is discussed. The model is developed by incorporating the various parametric values of MV network taken from electric supply companies in Pakistan, and is mainly comprised of three MV/LV transformers supplied from three-phase source. The suggested Simulink model contains the components of power system, designed to be operated at low power system frequency, i.e., $50 \mathrm{~Hz}$. However, it is significantly important to notice the role of transmitter and receiver blocks containing the CCD, as shown in Figures 7 and 8. Signal generator transmits the high frequency signal and injects in MV power line with the help of CCD thus the NB-PLC signal is imposed on $50 \mathrm{~Hz}$ power system. All three transformers have voltages of $11 \mathrm{kV}$ at MV side and $400 \mathrm{~V}$ at LV side, where LV network has $230 V_{L-N}$ and $400 V_{L-L}$. The power rating of first transformer is $150 \mathrm{kVA}$ with supply to the LV network of $140 \mathrm{~kW}$. The second transformer is rated at $250 \mathrm{kVA}$ supplying $245 \mathrm{~kW}$ to LV network. The third transformer is rated at $200 \mathrm{kVA}$ supplying $190 \mathrm{~kW}$ power to the LV network. The lengths of transmission line between source and 250 and $150 \mathrm{kVA}$ transformers are $1150 \mathrm{~m}$ and $950 \mathrm{~m}$, respectively, while there is a comparatively shorter, $110 \mathrm{~m}$ length between source and $200 \mathrm{kVA}$ transformer. In this model, the distributed parameter lines which are commonly used by electric supply companies in Pakistan are incorporated. These lines follow British Standard 215 with conductor type Wolf, Ant, and Panther. Table 2 tabulates the various values of parameters such as diameter, ampacity, resistance, capacitance, and inductance. The power transformers used in the simulation model are three phase, two winding transformers operating on power system frequency of $50 \mathrm{~Hz}$. Inductance and resistance are $0.50 \mathrm{H}$ and $5 \Omega$ for winding 1 , respectively, and $0.1 \mathrm{H}$ and $0.85 \Omega$ for winding 2 , respectively. The magnetization inductance $L_{m}$ and resistance $R_{m}$ of power transformers are $550 \mathrm{H}$ and $2 \mathrm{M} \Omega$, respectively, and $X / R$ ratio is 7 .

NB-PLC signal is injected and received with the help of CCD, at the same phase A of $250 \mathrm{kVA}$ transformer in MV power line i.e., at the MV side of transformer. The role of CCD is explained in the next subsection. The distance between transmitted and received signal is $1150 \mathrm{~m}$. It is worth noticing that NB-PLC signal can be injected and received in a similar way on the phases B and C. Furthermore, after a careful literature review, FS load of LV network is modeled as RLC load $[1,26]$. The ratios of active and reactive powers utilized in RLC loads of LV network, connected to MV network, are tabulated in Table 4.

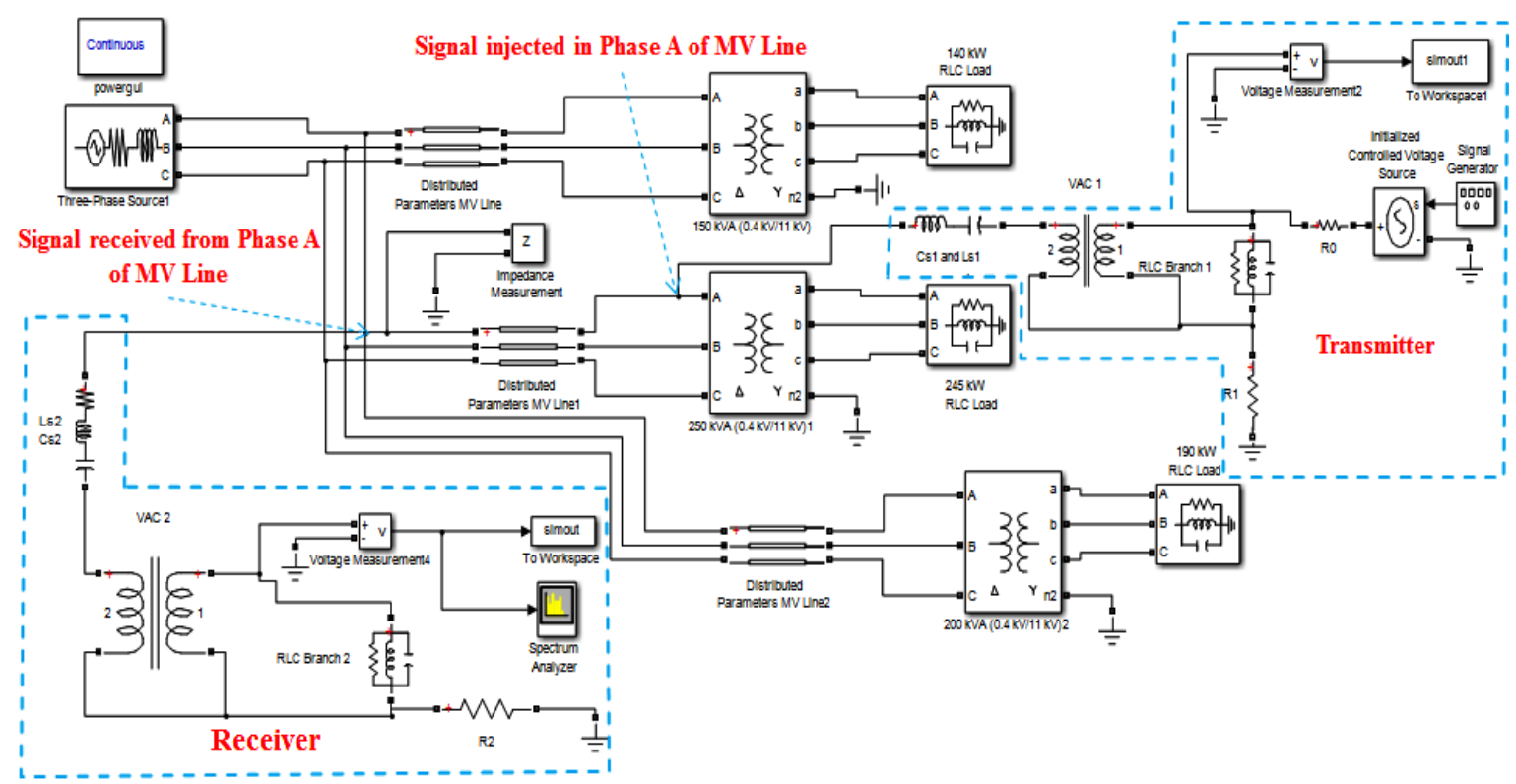

Figure 7. Proposed Simulink Model for MV NB-PLC channel modeling and characterization. 


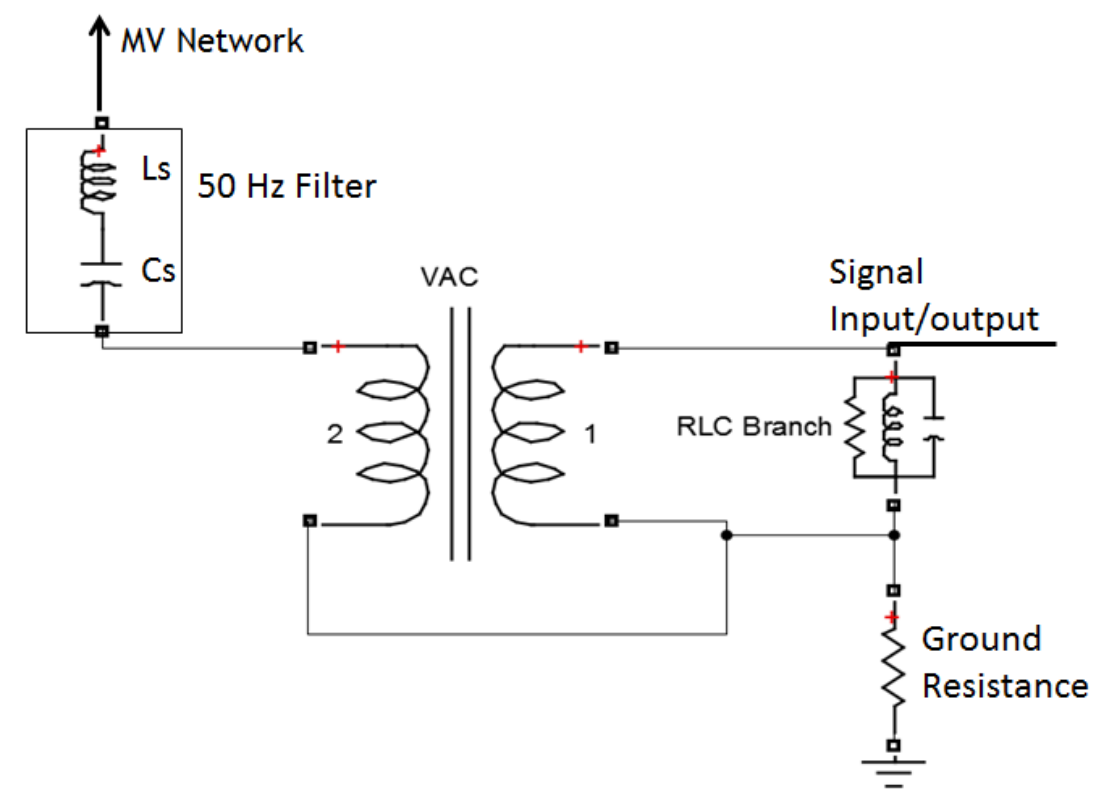

Figure 8. Circuit of MV phase to ground CCD.

Table 4. Active Power (W) and Reactive Power (VAR) ratios of LV network's RLC loads connected to MV network.

\begin{tabular}{cccc}
\hline & \multicolumn{2}{c}{ Active and Reactive Power Ratios of Connected RLC Load of LV Network } \\
\cline { 2 - 4 } Supplying Transformers & Active Power (kW) & \multicolumn{2}{c}{ Reactive Power } \\
\cline { 3 - 4 } & & $Q_{C}$ (+VAR) & $Q_{L}$ (-VAR) \\
\hline $150 \mathrm{kVA}$ & 140 & 30 & 85 \\
$200 \mathrm{kVA}$ & 190 & 20 & 95 \\
$250 \mathrm{kVA}$ & 245 & & 90 \\
\hline
\end{tabular}

\subsubsection{Capacitive Coupling Device}

A CCD is modeled to inject the signal in the MV power line for possible NB-PLC for AMI, without which the signal cannot be transmitted through an MV power line [36]. Figure 8 elucidates the schematic diagram of phase to ground CCD for MV network. A 1-V signal is generated with the help of a signal generator to the input of a parallel RLC circuit and further through isolation transformer tuned for the frequency range 3-500 kHz. The signal passes from a $50-\mathrm{Hz}$ filter before giving input to the MV power line. CCD is grounded with a resistance of $850 \Omega$. The values of various parameters used in CCD are tabulated in Table 5. The same CCD is used at the receiving end of MV NB-PLC system and on the transmitter side. Figure 9 illustrates the transfer function of CCD. The plotted results of CCD depicts the variations in attenuation between $10 \mathrm{~dB}$ and $-2 \mathrm{~dB}$.

Table 5. Capacitive coupling device parameters.

\begin{tabular}{cccccccc}
\hline \multicolumn{2}{c}{ Isolation Transformer } & & \multicolumn{2}{c}{ RLC Branch Parameters } & MV Series Ls Cs Parameters \\
\hline $\begin{array}{c}\text { Magnetization Resistance, } \\
\boldsymbol{R}_{\boldsymbol{M}} \boldsymbol{\Omega}\end{array}$ & Inductance, $L_{T}[\mu \boldsymbol{H}]$ & Turn Ratio & $\mathrm{R}[\mathrm{k} \Omega]$ & $\mathrm{L}[\mu \boldsymbol{H}]$ & $\mathrm{C}[\mathrm{nF}]$ & $L_{S}[\mu \mathrm{H}]$ & $C_{S} \mathrm{nF}$ \\
\hline 85 & 425 & $1: 1$ & 45 & 180 & 20 & 75 & 90 \\
\hline
\end{tabular}




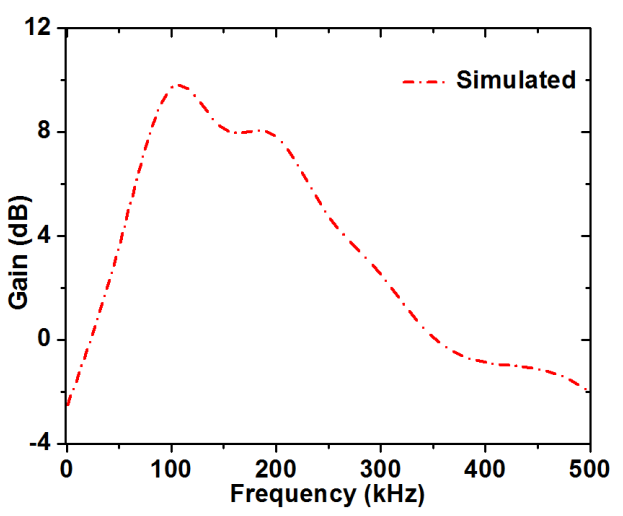

Figure 9. Simulated response of CCD.

\section{Results and Discussion of MV NB-PLC Channel Transfer Functions}

This paper proposes the channel modeling techniques for MV NB-PLC for AMI by comparing TL theory (with constant and FS impedances), multipath signal propagation model, and proposed Simulink model. This part of the paper discusses and compares the results of transfer functions determined from all suggested techniques.

\subsection{Transmission Line Theory Based Transfer Functions}

Figure 10a,b illustrates the transfer function results obtained from TL theory model by using constant and FS impedances of NB-PLC network, respectively. The attenuation profile of constant impedances-based transfer function is between -15 and $-40 \mathrm{~dB}$, whereas FS impedances-based transfer function varies between -11 and $-48 \mathrm{~dB}$. The FS impedances-based transfer function presents more peaks and dips as compared to constant impedances-based transfer function, e.g., peaks can be seen at 100, 250, and $355 \mathrm{kHz}$ and dips can be noticed at 75, 200, and $275 \mathrm{kHz}$ as well as a deep dip at $480 \mathrm{kHz}$.
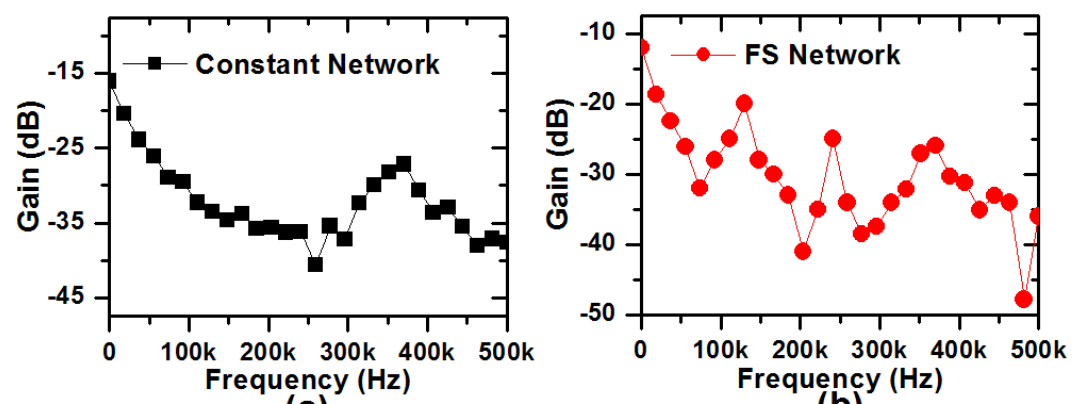

(a)

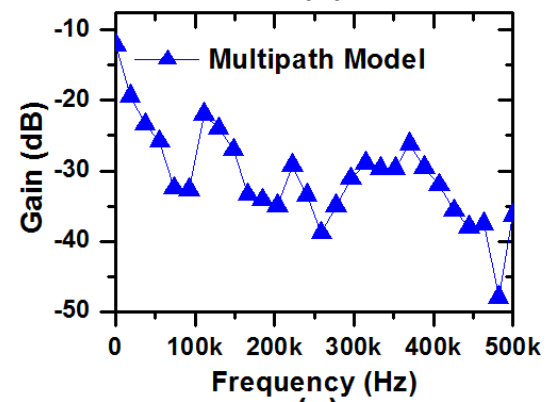

(c)

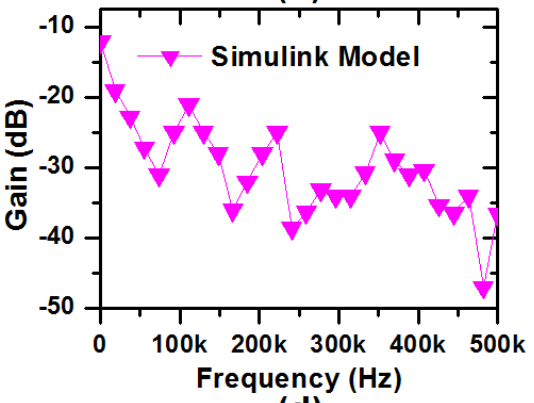

(d)

Figure 10. Transfer functions obtained from: (a) TL theory with constant network; (b) TL theory with frequency selective network; (c), ultipath signal propagation model; and (d) the proposed Simulink Model. 


\subsection{Multipath Signal Propagation Model Based Transfer Function}

Figure 10c shows the transfer function result obtained by implementing multipath signal propagation model on NB-PLC network. The attenuation profile is varying between -11 and $-44 \mathrm{~dB}$, illustrating peaks at 110, 220, and $370 \mathrm{kHz}$ and dips at 80-90, 200, 260, and $480 \mathrm{kHz}$.

\subsection{Proposed Simulink Model Based Transfer Function}

Figure $10 \mathrm{~d}$ presents the transfer function plot obtained by using the proposed Simulink model. The attenuation profile of transfer function is varying between -11 and $-45 \mathrm{~dB}$, illustrating peaks at 110,220 , and $350 \mathrm{kHz}$ and dips at 75, 170, 250, and $480 \mathrm{kHz}$.

It is clear from the plotted transfer function results that transfer function profile calculated from the proposed Simulink model follows the trend of transfer functions computed from FS-based TL theory and multipath signal propagation model, thus validating the performance of Simulink model. It is also noteworthy that constant impedances-based TL theory transfer function is comparatively more linear and does not give complete information about transfer function profile. However, FS-based transfer function computed by TL theory is more precise and follows the transfer function trends of multipath signal propagation and Simulink models.

\subsection{Box Plot Analysis for Attenuation Profiles}

A detail of attenuation profiles of transfer functions obtained from TL theory (constant and FS) model, multipath signal propagation model, and proposed Simulink model are segregated in different quartiles in the box plots shown in Figure 11. The frequency range of interest is $3-500 \mathrm{kHz}$ for NB-PLC network. The box plot consists of a type of plot able to visually reveal some basic statistics of an attenuation dataset by depicting the minimum values, maximum values, and trend of attenuation gains and drops. It is clear from the box plot analysis that attenuation profiles of all techniques are in good agreement with each other, except the attenuation profile obtained from constant impedances-based TL theory model. It is particularly significant to note that Simulink model provides an exhaustive set of information with wider means and extended quartiles.

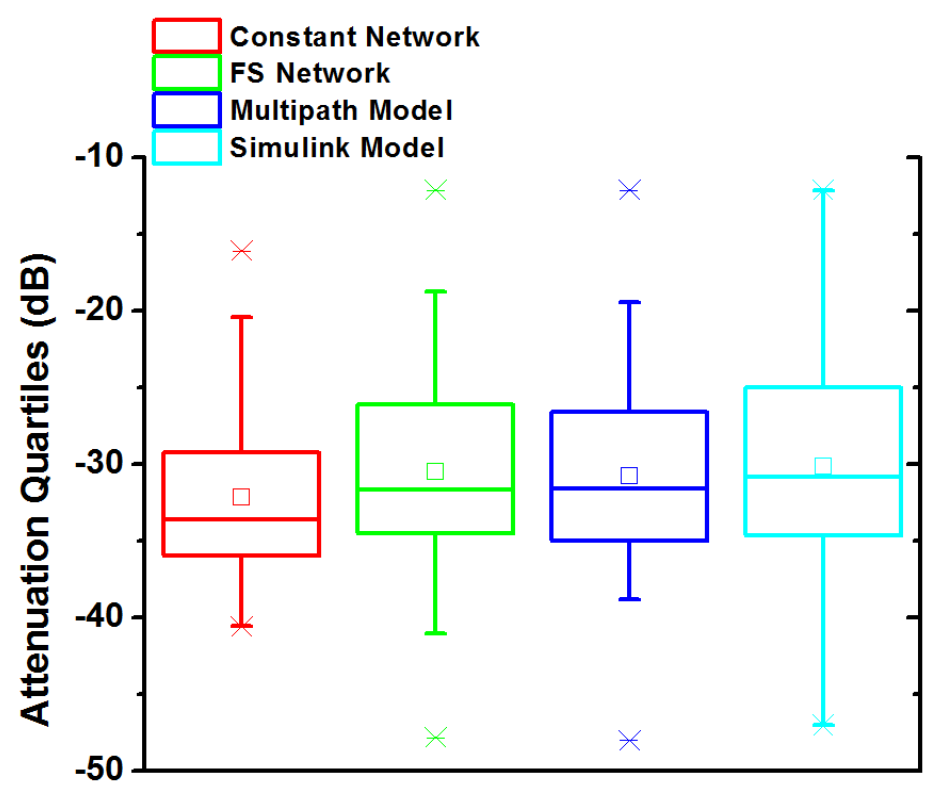

Figure 11. Summary of NB-PLC channels' transfer functions.

\section{Conclusions}

This paper presents the state of the art for NB-PLC channel modeling techniques for MV network by utilizing three different types of models for efficient channel modeling and characterization for 
MV NB-PLC network. The first technique is based on TL theory by considering the constant and FS parameters of transmission lines and load impedances. FS is introduced in the MV power lines to get a better estimate of channel conditions, leading to a scenario that is more closer to reality. The variations in the resistance values by adding frequency selectivity in it are compared with Resistance Variations law whose results are quite similar to each other. A statistical multipath signal propagation model is used that incorporates the effect of multipaths and reflections to compute the transfer function. After a careful investigation about the behavior of LV network, impedances are modeled and their corresponding plots with respect to increase in frequency are discussed. The third technique comprises of a Simulink model, developed by using real time parameters of power network. The CCD is modeled to inject the NB-PLC signal in MV network of Simulink model. The transfer functions of all techniques such as TL theory (constant and FS impedances) model, multipath signal propagation model, and proposed Simulink model are compared with each other. It is evident from the plotted transfer function results that FS transfer function of TL theory model and multipath signal propagation model are in good agreement and have similar trends of attenuation profiles with the transfer function computed from proposed Simulink model. In practical NB-PLC systems, network parameters and impedances do not remain constant but keep on changing for different values of frequencies. Therefore, close agreement of transfer function results of Simulink model with FS-based TL theory model and multipath signal propagation model validate the accuracy of Simulink model. The presented model in this paper can prove to be a useful simulation tool to estimate any MV NB-PLC channel conditions by simply changing the parametric values as per requirements and conditions.

Author Contributions: Conceptualization, B.M.; methodology, B.M. and M.A.K.; software, B.M.; formal analysis, B.M., G.S. and A.U.R.; writing-original draft preparation, B.M. and M.A.K.; writing—review and editing, B.M., S.B., A.U.R., S.U.R. and R.M.A.; supervision, B.M., M.B.R. and M.A.K.; project administration, B.M., M.B.R. and M.A.K.; and funding acquisition, B.M. and M.A.K. All authors have read and agreed to the published version of the manuscript.

Acknowledgments: This work was supported by the National Natural Science Foundation of China (NSFC) project (51950410583) and NSFC key project (U1766209).

Conflicts of Interest: The authors declare no conflict of interest.

\section{Abbreviations}

The following abbreviations are used in this manuscript:

$\begin{array}{ll}\text { AAC } & \text { All aluminium conductor } \\ \text { ACSR } & \text { Aluminium conductors steel reinforced } \\ \text { AMI } & \text { Advanced metering infrastructure } \\ \text { CCD } & \text { Capacitive coupling device } \\ \text { FS } & \text { Frequency selective } \\ \text { HDR } & \text { High data rate } \\ \text { LDR } & \text { Low data rate } \\ \text { LV } & \text { low voltage } \\ \text { MV } & \text { Medium voltage } \\ \text { NB-PLC } & \text { Narrowband power line communications } \\ \text { PLC } & \text { Power line communication } \\ \text { SG } & \text { Smart grid } \\ \text { TL } & \text { Transmission line }\end{array}$

\section{References}

1. Masood, B.; Baig, S. Standardization and deployment scenario of next generation NB-PLC technologies. Renew. Sustain. Energy Rev. 2016, 65, 1033-1047. [CrossRef]

2. Althaher, S.; Mancarella, P.; Mutale, J. Automated demand response from home energy management system under dynamic pricing and power and comfort constraints. IEEE Trans. Smart Grid 2015, 6, 1874-1883. [CrossRef] 
3. Goldfisher, S.; Tanabe, S. IEEE 1901 access system: An overview of its uniqueness and motivation. IEEE Commun. Mag. 2010, 48, 150-157. [CrossRef]

4. Smith, R.; Meng, K.; Dong, Z.; Simpson, R. Demand response: A strategy to address residential air-conditioning peak load in Australia. J. Mod. Power Syst. Clean Energy 2013, 1, 223-230. [CrossRef]

5. Yan, B.L.H.; Chen, S.; Zhong, M.; Li, D.; Jiang, L.; He, G. Future evolution of automated demand response system in smart grid for low-carbon economy. J. Mod. Power Syst. Clean Energy 2015, 3, 72-81. [CrossRef]

6. Masood, B.; Usman, M.; Gul, M.U.; Khan, W.A. Measurements and characterization of power transformer and low voltage access network for NB-PLC. Int. J. Commun. Syst. 2017, 30, e3344. [CrossRef]

7. Sharma, S.K.; Chandra, A.; Saad, M.; Lefebvre, S.; Asber, D.; Lenoir, L. Voltage flicker mitigation employing smart loads with high penetration of renewable energy in distribution systems. IEEE Trans. Sustain. Energy 2017, 8, 414-424. [CrossRef]

8. Gatsis, N.; Giannakis, G.B. Residential load control: Distributed scheduling and convergence with lost AMI messages. IEEE Trans. Smart Grid 2012, 3, 770-786. [CrossRef]

9. Zimmermann, M.; Dostert, K. A Multipath Model for the Powerline Channel. IEEE Trans. Commun. 2002, 50, 553-559. [CrossRef]

10. Zimmermann, M.; Dostert, K. Analysis and modeling of impulsive noise in broad-band powerline communications. IEEE Trans. Electromagn. Compat. 2002, 44, 249-258. [CrossRef]

11. Philipps, H. Modelling of Powerline Communication Channels. In Proceedings of the 3rd International Symposium on Powerline Communications and Its Applications, Lancaster, UK, 30 March-1 April 1999; pp. 14-21.

12. Matthias, G.; Rapp, M.; Dostert, K. Power line channel characteristics and their effect on communication system design. IEEE Commun. Mag. 2004, 42, 78-86.

13. Masood, B.; Usman, M.; Din, F.U.; Haider, A. Effect of transient and non-transient models on the performance of PLC. Telecommun. Syst. 2016, 65, 55-64. [CrossRef]

14. Masood, B.; Baig, S. Channel Modeling of NB-PLC for Smart Grid. In Proceedings of the 2015 IEEE Symposium on Computers and Communications (ISCC), Larnaca, Cyprus, 6-9 July 2015.

15. Banwell, T.; Galli, S. A Novel Approach to the Modeling of the Indoor Power Line Channel Part I: Circuit analysis and Companion Model. IEEE Trans. Power Deliv. 2005, 20, 655-663. [CrossRef]

16. Galli, S.; Banwell, T. A novel approach to the modeling of the indoor power line channel part II: Transfer function and its properties. IEEE Trans. Power Deliv. 2005, 20, 1869-1878. [CrossRef]

17. Sung, T.E.; Scaglione, A.; Galli, S. Time-Varying Power Line Block Transmission Models over Doubly Selective Channels. In Proceedings of the 2008 IEEE International Symposium on Power Line Communications and Its Applications, Jeju City, Korea, 2-4 April 2008.

18. Anatory, J.; Theethayi, N.; Thottappillil, R. Power-Line Communication Channel Model for Interconnected Networks-Part I: Two-Conductor System. IEEE Trans. Power Deliv. 2009, 24, 118-123. [CrossRef]

19. Anatory, J.; Theethayi, N.; Thottappillil, R. Power-Line Communication Channel Model for Interconnected Networks-Part II: Multiconductor System. IEEE Trans. Power Deliv. 2009, 24, 124-128. [CrossRef]

20. Tonello, A.M.; Versolatto, F. Bottom-Up Statistical PLC Channel Modeling-Part I: Random Topology Model and Efficient Transfer Function Computation. IEEE Trans. Power Deliv. 2011, 26, 891-898. [CrossRef]

21. Tonello, A.M.; Versolatto, F. Bottom-Up Statistical PLC Channel Modeling-Part II: Inferring the Statistics. IEEE Trans. Power Deliv. 2010, 25, 2356-2363. [CrossRef]

22. Tonello, A.M.; Versolatto, F. New Results on Top-down and Bottom-up Statistical PLC Channel Modeling. In Proceedings of the 2009 Third Workshop on Power Line Communications, Udine, Italy, 1-2 October 2009.

23. Cataliotti, A.; Cara, D.D.; Fiorelli, R.; Tine, G. Power-Line Communication in Medium-Voltage System: Simulation Model and Onfield Experimental Tests. IEEE Trans. Power Deliv. 2012, 27, 62-69. [CrossRef]

24. Artale, G.; Cataliotti, A.; Fiorelli, R.; Tine, G. Secondary substation power line communications for medium voltage smart grids. In Proceedings of the 2012 IEEE International Workshop on Applied Measurements for Power Systems (AMPS), Aachen, Germany, 26-28 September 2012.

25. Chu, G.; Li, J.; Liu, W. Narrow Band Power Line Channel Characteristics for Low Voltage Access Network in China. In Proceedings of the 2013 IEEE 17th International Symposium on Power Line Communications and Its Applications, Johannesburg, South Africa, 24-27 March 2013. 
26. Bausch, J.; Kistner, T.; Babic, M.; Dostert, K. Characteristics of indoor power line channels in the frequency range 50-500 kHz. In Proceedings of the 2006 IEEE International Symposium on Power Line Communications and Its Applications, Orlando, FL, USA, 26-29 March 2006.

27. Canete, F.J.; Cortes, J.A.; Diez, L.; Entrambasaguas, J.T. A channel model proposal for indoor Power Line Communications. IEEE Commun. Mag. 2011, 49, 166-174. [CrossRef]

28. Dong, L.; BaoHui, Z.; DongWen, N.; Bo, Z.Q.; Klimek, A. Design and implement of adaptive BFSK PLC system for medium voltage powerline communication. In Proceedings of the 45 th International Universities Power Engineering Conference UPEC2010, Cardiff, Wales, UK, 31 August-3 September 2010.

29. Cataliotti, A.; Cosentino, V.; Cara, D.D.; Tine, G. Oil-Filled MV/LV Power-Transformer Behavior in Narrow-Band Power-Line Communication Systems. IEEE Trans. Instrum. Meas. 2012, 61, 2642-2652. [CrossRef]

30. Cataliotti, A.; Cosentino, V.; Cara, D.D.; Tinè, G. Simulation and Laboratory Experimental Tests of a Line to Shield Medium-Voltage Power-Line Communication System. IEEE Trans. Power Deliv. 2011, $26,2011$. [CrossRef]

31. Masood, B.; Haider, A.; Baig, S. Modeling and Characterization of Low Voltage Access Network for Narrowband Powerline Communications. J. Electr. Eng. Technol. 2017, 12, 443-450. [CrossRef]

32. Masood, B.; Ellahi, M.; Khan, W.A.; Akram, W.; Usman, M.; Gul, M.T. Characterization and Field Measurements of NB-PLC for LV Network. J. Electr. Eng. Technol. 2018, 13, 521-531.

33. Cataliotti, A.; Daidone, A.; Tinè, G. A Medium-Voltage Cables Model for Power-Line Communication. IEEE Trans. Power Deliv. 2009, 24, 129-135. [CrossRef]

34. Benato, R.; Caldon, R. Frequency Characteristics Measurement of Overhead High-Voltage Power-Line in Low Radio-Frequency Range. IEEE Trans. Power Deliv. 2007, 22, 575-583. [CrossRef]

35. Kim, I.H.; Kim, W.; Park, B. Channel measurements and field tests of Narrowband Power Line Communication over Korean underground LV Power Lines. In Proceedings of the 18th IEEE International Symposium on Power Line Communications and Its Applications, Glasgow, UK, 30 March-2 April 2014.

36. Cataliotti, A.; Cosentino, V.; Cara, D.D.; Tine, G. Measurement issues for the characterization of medium voltage grids communications. IEEE Trans. Instrum. Meas. 2013, 62, 2185-2196. [CrossRef]

(C) 2020 by the authors. Licensee MDPI, Basel, Switzerland. This article is an open access article distributed under the terms and conditions of the Creative Commons Attribution (CC BY) license (http:/ / creativecommons.org/licenses/by/4.0/). 